\title{
STBCs with Optimal Diversity-Multiplexing Tradeoff for 2, 3 and 4 Transmit Antennas
}

\author{
V. Shashidhar, B. Sundar Rajan ${ }^{1}$ and P. Vijay Kumar ${ }^{1}$ \\ Dept. of Electrical Communication Engineering \\ Indian Institute of Science, Bangalore 560012, INDIA \\ e-mail: \{shashidhar@protocol., bsrajan,vijay\}@ece.iisc.ernet.in
}

\begin{abstract}
We show that the codes from division algebras [1] achieve the optimal diversity-multiplexing tradeoff for $n$ transmit and $n$ receive antennas for $n=$ 2,3,4 by simulation. Also, we present a lower bound for the tradeoff curve which shows that codes from division algebras for arbitrary number of transmit and receive antennas achieve points corresponding to zero diversity gain and zero multiplexing gain.

Let $S$ be the signal set over which we want to construct fullrank STBCs. Then, let $m$ be an integer such that $x^{n}-\omega_{m}$ is irreducible over $F=\mathbb{Q}\left(S, \omega_{m}\right)$, where $\mathbb{Q}$ is the field of rational numbers and $\omega_{m}=e^{j 2 \pi / m}$. The extension field obtained from this irreducible polynomial is $K=F\left(\omega_{m n}\right)$.
\end{abstract}

Theorem 1 ( [1]) Let $G$ be the Galois group of the extension $K / F$. Clearly, $G$ is cyclic and let $\sigma$ be a generator of this group. Let $\delta$ be a transcendental element over $F$. Then, the set $D$ of matrices of the form

$$
\left[\begin{array}{cccc}
\sum_{i=0}^{n-1} f_{0, i} t^{i} & \delta \sigma\left(\sum_{i=0}^{n-1} f_{n-1, i} t^{i}\right) & \cdots & \delta \sigma^{n-1}\left(\sum_{i=0}^{n-1} f_{1, i} t^{i}\right) \\
\sum_{i=0}^{n-1} f_{1, i} t^{i} & \sigma\left(\sum_{i=0}^{n-1} f_{0, i} t^{i}\right) & \cdots & \delta \sigma^{n-1}\left(\sum_{i=0}^{n-1} f_{2, i} t^{i}\right) \\
\vdots & \vdots & \ddots & \vdots \\
\sum_{i=0}^{n-1} f_{n-1, i} t^{i} & \sigma\left(\sum_{i=0}^{n-1} f_{n-2, i} t^{i}\right) & \cdots & \sigma^{n-1}\left(\sum_{i=0}^{n-1} f_{0, i} t^{i}\right)
\end{array}\right]
$$

where $t=\omega_{m n}$ and $f_{i, j} \in F(\delta)$ for $i, j=0,1, \ldots, n-1$ is a cyclic division algebra.

From the above theorem, restricting the variables $f_{i, j}$ to the signal set $S$ of size equal to $\mathrm{SNR}^{r / n}$, we have a full-rank STBC over $S$, for $n$ transmit antennas, with bit rate equal to $r \log \mathrm{SNR}$.

Example 1 (a) Let $n=2$ and $S$ be a $Q A M$ signal set. Then, we take $F=\mathbb{Q}(j), K=F(\sqrt{j})$. Let $\delta$ be any transcendental element over $F$. Then, we have a full-rank $S T B C \mathcal{C}(\mathrm{SNR})$ with codewords $\left[\begin{array}{cc}f_{0,0}+f_{0,1} \sqrt{j} & \delta\left(f_{1,0}-f_{1,1} \sqrt{j}\right) \\ f_{1,0}+f_{1,1} \sqrt{j} & \left(f_{0,0}-f_{0,1} \sqrt{j}\right)\end{array}\right]$ where $f_{i, j} \in$ $\mathrm{SNR}^{r / 2}-Q A M$.

(b) Let $n=3$ and $S$ be a QAM signal set. Then, we have a full-rank $S T B C \mathcal{C}(\mathrm{SNR})$ with codewords as in (1), where $\delta=e^{j 0.5}, t=\omega_{12}, \sigma(t)=t \omega_{3}$ and $f_{i, j} \in \mathrm{SNR}^{r / 3}-Q A M$.

(c) With $n=4$ and $S$ a $Q A M$ signal set, we have a fullrank $S T B C \mathcal{C}(\mathrm{SNR})$ with codewords as in (1), where $\delta=e^{j 0.5}$, $t=\omega_{16}, \sigma(t)=j t$ and $f_{i, j} \in \mathrm{SNR}^{r / 4}-Q A M$.

Theorem 2 The diversity-multiplexing tradeoff $d_{D A}(r)$ of the scheme $\{\mathcal{C}(\mathrm{SNR})\}$ obtained from division algebras for $Q A M$ and PSK signal sets satisfies

$$
d_{D A}(r) \geq 1-r / n \text { and } d_{D A}(0)=n^{2} .
$$

\footnotetext{
${ }^{1}$ This work was partly funded by the DRDO-IISc Program on Advanced Research in Mathematical Engineering through a grant to B.S.Rajan and by an NSF-ITR Grant 0326628 to P. Vijay Kumar.
}

Simulations: Figure 1(a) shows the error probability curves

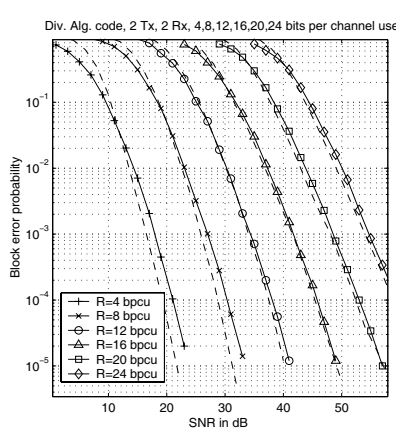

(a)

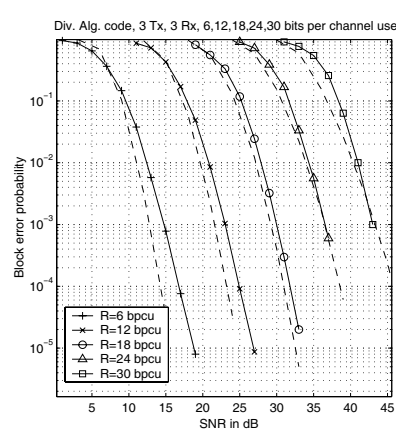

(b)
Figure 1: Error probability curves (solid) and outage probability curves (dashed): (a) 2 transmit and 2 receive antennas, (b) 3 transmit and 3 receive antennas.

for various data rates, for 2 transmit and 2 receive antennas. It can be seen that at high SNRs, the gap between two adjacent curves, with data rates differing by 4 bits per channel use, is 6 $\mathrm{dB}$. This indicates that at $d=0$, the data rate grows with SNR as $R=2 \log$ SNR. Thus, the point $(2,0)$ of the tradeoff curve is achieved. We have also plotted the outage probabilities (dashed curves). It can be seen that curves for $P_{e}$ match with outage probability at high SNRs and hence the DA code for 2 transmit and 2 receive antennas achieves the optimal tradeoff. Similar conclusions can be drawn for $n=3$ and $n=4$ cases of Example 1 from Figures 1(b) and 2 respectively.

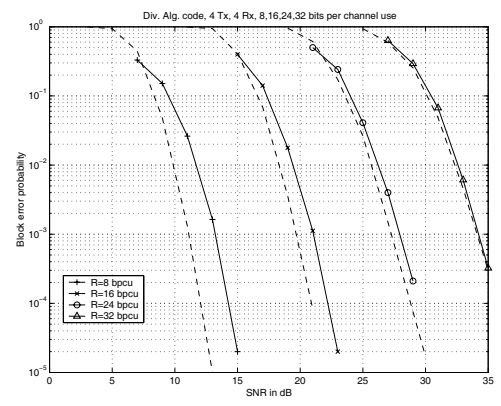

Figure 2: Error probability curves (solid) and outage probability curves (dashed)for 4 transmit and 4 receive antennas.

\section{REFERENCES}

[1] B. Sethuraman, B. Sundar Rajan and V. Shashidhar, "Fulldiversity, high-rate space-time block codes from division algebras," IEEE Trans. Inform. Theory, vol. 49, no. 10, pp. 25962616, Oct. 2003.

[2] LiZhong Zheng, David N. C. Tse, "Diversity and multiplexing: A fundamental tradeoff in multiple-antenna channels", IEEE Trans. Inform. Theory, vol.49, no.5, pp.1073-1096, May 2003. 